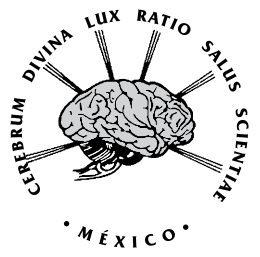

\title{
Análisis del factor de impacto de la Revista Enfermería Universitaria de la ENEO-UNAM 2011
}

\section{ENEO-UNAM'S Enfermería Universitaria Journal impact factor analysis 2011}

\author{
Lucía Fabiola Martínez-Fajardo, ${ }^{*}$ Lasty Balseiro Almario, ${ }^{* *}$ Federico Sacristan Ruiz, ${ }^{\star * *}$ \\ Gandhy Ponce Gómez, ${ }^{* * *}$ Silvia Hernández Rosas, ${ }^{* * *}$ Francisco Blas Valencia**** \\ * Pasante de Servicio Social de la Licenciatura en Enfermería. ENEO-UNAM. México. \\ ** Responsable del Proyecto. Unidad de Investigación de la ENEO-UNAM. México. \\ *** Corresponsable del Proyecto. Sistema de Universidad Abierta y Educación Continua de la ENEO-UNAM. México. \\ $\star * * *$ Colaborador del Proyecto.
}

\begin{tabular}{|c|c|}
\hline \multicolumn{2}{|c|}{ RESUMEN } \\
\hline $\begin{array}{r}\text { Palabras clave: } \\
\text { Factor de impacto, } \\
\text { revista, Enfermería } \\
\text { Universitaria, } \\
\text { estudio } \\
\text { bibliométrico, } \\
\text { artículos } \\
\text { publicados, citas. }\end{array}$ & $\begin{array}{l}\text { El objeto de esta investigación fue identificar el factor de impacto de la Revista Enfermería Universitaria de la ENEO en el año } 2011 \text {, } \\
\text { como parte de la evaluación interna de la revista. Marco teórico: El factor de impacto de las revistas es el índice bibliométrico más } \\
\text { utilizado para evaluar el número de citas que reciben en promedio y los trabajos publicados en una determinada revista. El hecho } \\
\text { de publicar en revistas de alto factor de impacto se considera, en muchos contextos, un criterio de calidad en sí mismo, pues se } \\
\text { asume que un artículo publicado en determinada revista es probable que reciba el mismo número de citas que el promedio de la } \\
\text { revista. Metodología: Es un estudio bibliométrico, de corte cuantitativo, no experimental, longitudinal, retrospectivo, descriptivo y } \\
\text { diagnóstico. La población de estudio la constituyen } 47 \text { artículos originales y de revisión, publicados en la revista en los años } 2009 \text { y } \\
2010 \text {. Se utilizan métodos cuantitativos para estudiar la citación y métodos cualitativos para realizar el análisis del factor de impacto. } \\
\text { La técnica de recolección de datos se realizó mediante una revisión manual de todos los artículos publicados, estandarizando el } \\
\text { método de revisión de la revista. Resultados: En los tres años estudiados, se publicaron } 13 \text { números de la revista con } 34 \text { artículos } \\
\text { de investigación y } 13 \text { de revisión, dando un total de } 47 \text { artículos con ocho citas entre } 2009 \text { y } 2010 \text {, lo que da un factor de impacto de } \\
0.1702 \text { para } 2011 \text {. Conclusiones: El factor de impacto de la revista ha ido en incremento, lo que implica que está siendo emisora y } \\
\text { receptora de citas en las investigaciones publicadas al } 2011 \text {. Sin embargo, es necesario incrementar el factor de impacto de la Revista } \\
\text { Enfermería Universitaria, lo que redundará en la calidad y la importancia de los grupos de investigación que en ella publican. }\end{array}$ \\
\hline \multicolumn{2}{|c|}{ ABSTRACT } \\
\hline $\begin{array}{r}\text { Key words: } \\
\text { Impact factor, } \\
\text { journal, } \\
\text { University } \\
\text { Nursing, } \\
\text { journal-metric } \\
\text { study, published } \\
\text { articles, citations. }\end{array}$ & $\begin{array}{l}\text { The aim of this research was to address the impact factor of the ENEO's Enfermería Universitaria Journal during the year 2011, } \\
\text { as part of its internal assessment process. Theoretical framework: The Impact Factor of journals is the most widely use index } \\
\text { to estimate the average number of citations and works involved. Publishing in journals with a high Impact Factor is considered, } \\
\text { in various contexts, a quality criterion since it is assumed that an article published in a determined journal will receive the } \\
\text { same average number of citations as the journal itself. Methodology: This is a quantitative, not experimental, Iongitudinal, } \\
\text { retrospective, descriptive, and diagnostic study. The population is made of } 47 \text { original and revision articles published in the } \\
\text { journal during the years } 2009 \text { to } 2010 \text {. Quantitative and qualitative methods are used to study the citation average numbers as } \\
\text { well as the Impact Factor. Data collection was accomplished through a standardized manual revision of all published articles. } \\
\text { Results: During the three years studied, } 13 \text { issues with } 34 \text { research and } 13 \text { revision articles were published, } 47 \text { articles had } \\
\text { eight citations between } 2009 \text { and } 2010 \text {, yielding impact factors of } 0.1702 \text { in } 2011 \text {. Conclusions: The Journal's Impact Factor } \\
\text { has had a steady growth; however it is necessary to increment it even more, in order to emphasize importance of the journal's } \\
\text { research groups works. }\end{array}$ \\
\hline
\end{tabular}

Recibido para publicación: 03/09/2014. Aceptado: 11/09/2014.

Este artículo puede ser consultado en versión completa en: www.medigraphic.com/enfermerianeurologica 


\section{INTRODUCCIÓN}

El problema planteado fue identificar ¿cuál es el factor de impacto (FI) de la Revista Enfermería Universitaria 2011? El objetivo fue analizar las citas y los artículos de investigación y revisión que permitan valorar el Factor de Impacto en el periodo estudiado. Marco teórico: Para Lorenzini y cols., ${ }^{1}$ el FI comenzó a ser considerado como una manera de evaluar las revistas científicas, a partir de la década de los 60, siglo XX, cuando Eugene Garfield, director del Institute of Scientific Information (ISI) y creador de la base de datos bibliográfica Science Citation Index (SCI), eligió ese instrumento que determina la frecuencia con que un artículo es citado, como forma de clasificar y evaluar las revistas incluidas en la referida base de datos. Solamente los periódicos indexados en el ISI son considerados para el cálculo del FI internacional. El FI es divulgado por el Journal Citation Report (JCR). De hecho, para Téllez y cols. ${ }^{2}$ el FI lo determina el Instituto de Información Científica (ISIsiglas en inglés - Ilamado actualmente Thomson-ISI). EI Thomson-ISI fue fundado en 1958, con la función de dar a los investigadores un acceso a la información relacionada con la investigación científica de alta calidad. Entonces, según Orts, ${ }^{3}$ el $\mathrm{FI}$ de una revista se puede definir como el número de citas que, por término medio, ha recibido el conjunto de los artículos recientes publicados por la misma. Se entiende por recientes, los correspondientes a los dos últimos años. Es decir, es la media de citas que recibe una revista por artículo publicado. Según Amezcua, ${ }^{4}$ los índices de impacto (II) son indicadores bibliométricos que pretenden clasificar las revistas en función de su repercusión en la comunidad científica. Y ello lo hacen midiendo el número de citas que reciben los artículos durante un periodo determinado de tiempo o a partir de su publicación. Según Camps, ${ }^{5}$ entre los indicadores que utiliza el ISI pueden mencionarse, entre otros, el Índice de Inmediatez, número de artículos por revista, número de artículos por autor, vida media de las citas, índice de colaboración, número de artículos por categoría, número de revistas por categoría. La fórmula utilizada por el ISI para obtener el FI de una revista es el número de citaciones realizadas a artículos publicados en una revista en un periodo determinado (citaciones hechas por distintas revistas indexadas en el ISI) dividido por el total de artículos de esa revista. Según Ruiz y cols., ${ }^{6}$ para calcular el FI estándar es de común conocimiento que se trata del coeficiente promedio resultante de dividir las veces que los artículos recientes (publicados en los dos últimos años) de una revista son citados en un año concreto (el del cálculo), entre el número de artículos publicados en el mismo periodo. Bordons y Zulueta ${ }^{7}$ destacan, como importante, que impacto y calidad de la investigación no son sinónimos. El término calidad se refiere al contenido científico de la publicación, a lo adecuado de la metodología, a la claridad de la exposición y la originalidad de planteamientos y conclusiones. El impacto, por su parte, se refiere a la influencia de la publicación sobre la investigación afín en un momento determinado. Entonces, para Gómez y Bordons ${ }^{8}$ el hecho de publicar en revistas de alto $\mathrm{FI}$ se considera, en muchos contextos, un criterio de calidad en sí mismo, pues se asume que un artículo publicado en determinada revista es probable que reciba el mismo número de citas que el promedio de la revista. Para Gálvez Toro, ${ }^{9}$ en la ciencia todos los grupos socialmente definidos tienden a citarse endogámicamente. Aunque esto se produce con diversa intensidad, el conocimiento proveniente de las ciencias básicas fluye de forma más universal que el de las ciencias aplicadas; igual ocurre con las ciencias biomédicas y en general las ligadas a las tecnologías en relación con las ciencias sociales y las humanidades. Analizar a la ciencia como un fenómeno unitario conlleva desajustes inevitables, favoreciendo a los sectores socialmente hegemónicos en detrimento del resto, que quedaría relegada a posiciones marginales. La enfermería iberoamericana es doblemente marginada por la ciencia hegemónica: por ser enfermería y por no expresarse en inglés. Así, Téllez y cols. señalan que el FI de las revistas latinoamericanas es bajo, y esto podría considerarse una mala noticia para sus editores. Sin embargo, la mayoría de los editores regionales pasan a un segundo término el FI y defienden el papel de cada una de estas revistas como uno de los pocos o el único medio de difusión del conocimiento médico en sus propios países. Amezcua opina que en el campo de la enfermería cabe esperar que una revista obtenga mayor impacto si es analizada junto con otras revistas de enfermería, más que si lo es junto a revistas médicas. Esto es así porque las enfermeras tienden a leer y citar lo que otras enfermeras publican: Por ejemplo, las enfermeras geriátricas tienden a leer y citar lo que otras enfermeras geriátricas producen. Las enfermeras anglosajonas tienden a citarse entre ellas, mientras que las enfermeras brasileñas citan principalmente artículos que publican las revistas de su país. No obstante, Gálvez, ${ }^{10}$ considerando el efecto de las autocitas sobre la repercusión, observa un fenómeno curioso: las revistas especializadas y las revistas latinoamericanas tienden a realizar más autocitas que la media. Esto significa que la comunidad de enfermeras que publica en ellas tiende a utilizar como referente bibliográfico de primer orden lo ya publicado en estas revistas. Esto, en cierto modo, denota un aislamiento en el consumo de información, ya que los autores citan la misma revista en la que publican, llegando a darse el caso extremo de algunas revistas que sólo reciben citas de sí mismas. En cuanto a las limitaciones del $\mathrm{FI}$, Gálvez ${ }^{11}$ dice que Bravo y Ferreiro, al igual que López, Piñero y Terrada, las explican con precisión, entre las que destacan dos: Es un indicador relativo y no es útil para establecer, por sí solo, criterios sólidos de evaluación. Para Fernández y cols. ${ }^{12}$ tales críticas alcanzan a los autores que intentan publicar en revistas con $\mathrm{FI}$, argumentando que así renuncian a la propia identidad, privando su currículum sobre la utilidad de su investigación. En oposición a esas críticas se afirma que unos criterios de evaluación exigentes sirven de estímulo para la internacionalización del sistema científico. A pesar de ello, existe consenso en la comunidad académica sobre las imperfecciones del FI y su aceptación como recurso válido y necesario para la 
Enf Neurol (Mex) • 2014 • Vol. 13 • Núm. 3 • 107-111

evaluación científica, aunque contribuye poco a resolver la invisibilidad internacional de la investigación en enfermería en español.

\section{MATERIAL Y MÉTODOS}

La variable medida fue el FI de la revista. Los indicadores fueron las citas y los artículos producidos en el periodo 2008 y 2009 para analizar el FI del 2011 y que formaron parte de la evaluación interna de la Revista. El diseño metodológico fue de corte cuantitativo, bibliométrico, no experimental, longitudinal, retrospectivo y descriptivo. La población de estudio fueron 65 artículos publicados en la Revista, de los cuales se incluyeron como muestra, por conveniencia, 34 artículos de investigación y 13 de revisión, dando un total de 47. Para el análisis de citación se revisaron las referencias que los investigadores consultan y emiten en sus investigaciones. La técnica de recolección de datos se realizó mediante una revisión manual de los artículos publicados, y el registro de los datos en cuadros de frecuencia acumulada. Se excluyeron los artículos editoriales, reseñas, ensayos, de opinión y estudios de caso. Se utilizaron métodos cuantitativos para el recuento y métodos cualitativos para el análisis de las citaciones. Para el proceso de evaluación interna, se utilizaron las técnicas de observación y análisis de las citaciones y se dividió el resultado entre el número de artículos publicados de investigación, a partir de la emisión de las citas en el periodo analizado. La fórmula para realizar la medición del FI fue: $\mathrm{FI}=$ No. de citas recibidas/No. de artículos publicados. En las consideraciones éticas no existió ningún riesgo para los pares y se tomó en cuenta el principio de anonimato y privacidad, conforme al reglamento de la Ley General de Salud que se refiere al principio de la privacidad, en donde prevalece el criterio de respeto a la dignidad y a la protección de los derechos y bienestar de los seres humanos.

\section{RESULTADOS}

La Revista Enfermería Universitaria publicó del 2009 al 2011, 65 artículos de diversos tipos: originales, de revisión, ensayos, de opinión, editoriales, estudios de caso y de práctica profesional, entre otros. En materia de artículos de revisión y originales, hasta el 2010, se publicaron 34 artículos de investigación que representan un 52.30\% y 13 de revisión, con $20.00 \%$. Para el periodo 2009-2010 se produjeron 34 artículos de investigación y 13 de revisión dando un total de 47 (Cuadro I). En cuanto a las citas

\begin{tabular}{|lrcc|}
\hline \multicolumn{4}{c}{ Cuadro I. Artículos 2009-2010. } \\
\hline Tipos de artículos & 2009 & 2010 & Totales \\
\hline Investigación & 18 & 16 & 34 \\
Revisión & 5 & 8 & 13 \\
\hline Totales & 23 & 24 & 47 \\
\hline
\end{tabular}

registradas, fueron ocho de investigación y ninguna de revisión, con un total de ocho citas (Cuadro II). Esto produjo para 2009-2010, ocho citas recibidas y 47 artículos (Cuadro III). Al realizar la división entre citas y artículos, dio un FI de 0.1702 para 2011 (Cuadro IV). Los artículos que recibieron las citas son: "Enseñanza clínica en el laboratorio: un acercamiento desde el discurso docente", "Seguimiento de egresados de la Lic. de la ENEO: formación y desempeño laboral 2003-2006", "Salud mental del adulto mayor", "Calidad de vida de los adultos mayores y su percepción del cuidado de enfermería", "Calidad del cuidado y satisfacción de la persona y Perspectiva bioética en el cuidado de enfermería".

\section{DISCUSIÓN}

La producción científica, en términos bibliométricos, es la suma de los productos científicos individualizados generados por una determinada comunidad (nacional, internacional, sectorial, etc.) durante un periodo convenido, y físicamente identificables como publicaciones, cualquiera que sea su forma. Una herramienta utilizada para evaluar dicha producción científica, y más concretamente el estudio de las publicaciones científicas, es la bibliometría. Los

\begin{tabular}{|lccc|}
\hline \multicolumn{4}{c}{ Cuadro II. Citas 2009-2010. } \\
\hline Citas recibidas & 2009 & 2010 & Totales \\
\hline Investigación & 4 & 4 & 8 \\
Revisión & 0 & 0 & 0 \\
Totales & 4 & 4 & 8 \\
\hline
\end{tabular}

\begin{tabular}{|lcc|}
\hline \multicolumn{3}{|c|}{ Cuadro III. Artículos y citas 2009-2010. } \\
\hline Año & Citas recibidas & Artículos \\
\hline 2009 & 4 & 23 \\
2010 & 4 & 24 \\
Totales & 8 & 47 \\
\hline
\end{tabular}

\section{Cuadro IV. Factor de impacto 2011.}

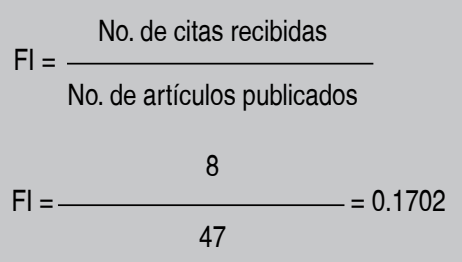

$\mathrm{FI}=0.1702$ 
indicadores bibliométricos más utilizados para medir la actividad científica se basan en los recuentos de las publicaciones y de las citas recibidas por los trabajos publicados. De acuerdo con Bordons y Zulueta, el FI de las revistas es un indicador presente en casi todos los estudios bibliométricos y mide específicamente la visibilidad y la difusión de los trabajos publicados en revistas más que la calidad científica de los mismos. El análisis de citas conlleva el recuento del número de citas que reciben los documentos durante un determinado periodo de tiempo después de su publicación. En este contexto, en la Revista Enfermería Universitaria se han analizado las citas en el periodo 2009-2010, logrando FI de 0.1702 para 2011, lo que permite identificar que el FI de la revista, aunque es bajo, en un rango de $0-3$, en virtud de las citaciones, la calidad de los artículos citados es buena y ha mejorado de un periodo a otro, lo que muestra mayor visibilidad de la Revista en el ámbito local y nacional en México. A nivel Latinoamericano, en un estudio sobre la actividad científica, Gálvez et $\mathrm{al}^{13}$ señalan que el ISI publicó en su sección de indicadores regionales, en marzo de 2004, el ranking de las seis instituciones educativas latinoamericanas más citadas entre 1992 y junio 2003 en índices de corriente principal; en éste se incluye a Brasil, con las universidades de Sao Paulo, Estatal de Campiñas y Federal de Río de Janeiro; a México, con la Universidad Nacional Autónoma de México; a Argentina, con la Universidad de Buenos Aires; y a Chile, con la Universidad Católica de Chile. En conjunto, sus artículos han sido citados más de 200,000 veces en ese periodo. Los resultados obtenidos en este estudio son compatibles con los hallazgos reportados en estudios del Grupo de Estudios Documentales ${ }^{14}$ en 2002, que considera que hay un conjunto de revistas de enfermería que son más citadas que las restantes y, de entre ellas, destacan varios títulos por su repercusión y visibilidad en el conjunto de la comunidad científica del área lingüística del español y del portugués. Por su inmediatez, las revistas con mayor repercusión son: Index de Enfermería con FI de 0.80, Enfermería Clínica con 0.73, Enfermería Intensiva (España) con 0.69, Desarrollo Científico de Enfermería (México) con 0.40, Revista Brasileira de Enfermagem (Brasil) con 0.35, Matronas Profesión con 0.32, Rol de Enfermería (España) con 0.31, Revista Latinoamericana de Enfermagem con 0.31 (Brasil) y Gerokomos (España) con 0.25. La Fundación Index, ${ }^{15}$ de Granada, España, en los últimos estudios bibliométricos ha podido identificar revistas de siete países diferentes. Aunque con unos indicadores de impacto desiguales a nivel nacional e internacional, las principales revistas con repercusión visible son: Index Enfermería (España), Investigación y Educación en Enfermería (Colombia), Escola Anna Nery Revista de Enfermagem (Brasil), Revista Latinoamericana de Enfermagem (Brasil), Enfermería Intensiva (España), Revista Rol de Enfermería (España), Cultura de los Cuidados (España), Ciencia y Enfermería (Chile). En España, las revistas más visibles son: Index de Enfermería, Enfermería Clínica, Rol de Enfermería, Enfermería Intensiva. Este núcleo, sin embargo, se ve modificado cada año cuando el GED actualiza los datos.
La repercusión y el impacto de una revista son indicadores vivos y de actualidad. Así, en una muestra de 12 revistas en España, aquellas con mayor $\mathrm{FI}$, se incluyan o no las autocitas, son: Rol de Enfermería (3.39), Enfermería Clínica (2.52), Gerokomos (2.27), Index de Enfermería (1.21), Ilíades (1.19) y Enfermería Científica (1.10). Las restantes tienen un FI por debajo de 1 . Si se comparan los resultados del FI de Enfermería Universitaria con los resultados de las revistas de Enfermería Españolas, éstas superan a Enfermería Universitaria; sin embargo, son iguales en las cifras superiores a 0 . Esto significa que Enfermería Universitaria tiene, como revista científica, repercusión visible en español, en México, considerando que la revista se empieza a publicar, según Balseiro et al, ${ }^{16}$ en 2004 , y alcanza mayor visibilidad en 2011, con mejores indicadores de visibilidad e impacto. De hecho, el incremento en la producción bibliográfica de la Revista y la creciente necesidad de conocer la documentación existente hacen de las bases de datos una de las herramientas más útiles en la recuperación selectiva de la información bibliográfica y de la citación. A este respecto, Sobrido ${ }^{17}$ considera: "si comparamos el FI de las revistas españolas de Enfermería con los datos hasta ahora facilitados podemos observar cómo todas las revistas con $\mathrm{FI}$ son recogidas en las bases de datos Cuiden, Cuidatge y Enfispo. En cuanto a las bases de datos internacionales, todas las revistas que han sido indizadas contienen Fl". A pesar de ello, Torra i Bou, ${ }^{18}$ quien analiza tres publicaciones enfermeras en el trienio 1991-1993, y Gálvez Toro y cols., basándose en el análisis de referencias y de citas, llegan a la conclusión de que las enfermeras consultan pocas revistas de Enfermería al escribir sus artículos. Es así que Fernánde $z^{19}$ plantea que ante la importancia que adquiere el FI en la evaluación de la actividad investigadora, en primer lugar tendemos a publicar nuestros artículos en revistas con impacto, con lo que las publicaciones de enfermería no incluidas corren el riesgo de quedar relegadas. Pero, sin renunciar a publicar nuestras investigaciones en las revistas de mayor impacto, cabe desarrollar una doble estrategia: publicar en ambas (con y sin FI) esforzándose por incluir y mantener a las revistas de enfermería en español mejor situadas.

\section{CONCLUSIONES}

Las revistas científicas desempeñan un importante papel en el proceso de comunicación científica, ya que constituyen la principal vía para la validación del nuevo conocimiento y hacen posible su difusión dentro de la comunidad de investigadores. El FI es un indicador bibliométrico que permite medir la visibilidad y repercusión de la publicación a partir del número de citas que recibe la revista entre la producción de artículos en un tiempo determinado. Las revistas con alto $\mathrm{FI}$, reúnen los mayores criterios de calidad, prestigio y difusión. Así, las citas y referencias constituyen un aspecto de gran importancia en las publicaciones científicas, ya que son instrumentos de evaluación de la calidad de la revista. En la Revista Enfermería Universitaria, desde su creación, por primera vez se realiza el ejer- 
cicio de medir el FI, que aunque es bajo, es superior a 0 , lo que muestra que ya empiezan a citar los autores las publicaciones de la revista. El incremento observado de 0.1702 en 2011 así lo demuestra. Medir el FI de la Revista ha puesto de manifiesto también que falta publicar más $y$, de igual forma, consumir la información que las enfermeras publican. Además, la revista carece de autocitas, ya que los investigadores que escriben reúnen citas, en muchos de los casos, internacionales. El intercambio de citas es necesario, pero también que las Revistas estén incluidas en bases de datos nacionales e internacionales, para facilitar el proceso de medición de los indicadores bibliométricos, la difusión de los artículos y del camino de la información científica.

\section{BIBLIOGRAFÍA}

1. Lorenzini RA, Palucci MM, Songalves PM, Feliz LF, Freitas PL, Itayra PM et al. La evaluación de periódicos científicos Qualis y la producción Brasileña de artículos del área de enfermería. Rev Latino-am Enfermagem [Internet]. 2009 maio-junho; 17(3): 1-8, Disponible en: www.eerp.usp.br/rlae.

2. Téllez ZJF, Morales BLE, Estañol B. Análisis del factor de impacto de las revistas científicas latinoamericanas. Rev Méd Chile. 2007; 137: 480-487.

3. Orts CM, Richart MM, Cabrero GJ. Factor de impacto en las revistas de enfermería. Enfermería Clínica. 2002; 12 (6): 266272.

4. Amezcua M. ¿Para qué sirve el índice de impacto de una revista? Index de Enfermería [Internet]. $2010 \mathrm{abr}$ sep 19(2): 1-11. [Consultado el 19 de agosto 2011]. Disponible en: http://scielo.iscii.es/scielo.php?pid=51132$12962010000200002 \&$ script.sciarllext\&tlng $=p t$

5. Camps D. Limitaciones de los indicadores bibliométricos en la evaluación de la actividad científica biomédica. Colombia Médica. 2008; 39 (1): 74-79.

6. Ruiz PR, Delgado LE, Jiménez CE. Criterios del Institute for Scientific Information para la selección de revistas científicas. Su aplicación a la Revista española: metodología e indicadores. International Journal of Clinical and Health Psychology [Internet]. 2006; 6 (2): 401-424. [Consultado el 19 de agosto 2011]. Disponible en: http://redalyc.uaemex.mx

7. Bordons M, Zulueta A. Evaluación de la actividad científica a través de los indicadores bibliométricos. Española de Cardiología. 1999; 52: 790-800.

8. Gómez Cl, Bordons SM. Limitaciones en el uso de los indicadores bibliométricos para la evaluación científica. Centro de información y documentación científica CINDOC, CSIC. Universidad de Alcalá. Madrid, 1999: 21-26.

9. Gálvez TA. El Español proscrito. Evidentia. 2007; 4(15). En Amezcua M. ¿Para qué sirve el Índice de Impacto de una Revista? Index de Enfermería [Internet]. 2010; 19 (23): 1-11. Disponible en: http://www.index-f.com/evidentia/ n15/34articulo.php

10. Gálvez TA. Hacia la universalización de enfermería en el área lingüística del español y portugués. Desarrollo científico de Enfermería. 2004; 12 (3): 67-79.

11. Gálvez TA, López MI, Sánchez CV y Poyatos HE. Impacto y aislamiento en el año 2000. Index de Enfermería. 2001; (34): 54-64.

12. Fernández SC, Granero MS, Hernández PJ y Aguilera MG. Factor de impacto de revistas: amenaza y oportunidad. Chía. 2011; 11 (3): 245-255.

13. Gálvez TA, López MM, Sánchez CV, Poyatri HE. Evaluación de la actividad científica de la Enfermería Española: Impacto y asilamiento en el año 2000. Index de Enfermería. Granada, 2001; 10 (34): 54-64.

14. Gálvez TA, Hueso MC, Amezcua M. Indicadores CUIDEN de repercusión en las revistas de enfermería: Del área lingüística del español y del portugués. Index de Enfermería. Granada, 2004; 46: 76-80.

15. Fundación Index. Información bibliográfica y documental. Grupo de Estudios Documentales. Granada, 2004: 2-4.

16. Balseiro L, Torres MA y Zárate RA. Evaluación interna y externa de la Revista Enfermería Universitaria de la ENEOUNAM, durante el periodo 2004-2006. Enfermería Universitaria. México. 2009; 6 (1): 12-21.

17. Sobrido SP, Sobrido PN, González GC, Pichel GM, García SM, Prieto DA. Revistas Españolas de Enfermería en bases de datos nacionales e internacionales. Index de Enfermería. Granada, 2005; XIV (48-49): 74-77.

18. Serrano SP, Jiménez MA, Arroyo GM. Análisis de la producción científica en la revista Métodos de Enfermería. Informe Especial. Index de Enfermería. Granada. 2005; XIV (48-49): 78-82.

19. Fernández SC, Granero MJ, Hernández PJM, Aguilera MG. Factor de impacto de revistas: ¿amenaza u oportunidad? Aquichán. 2011; 11 (3): 245-255.

Dirección para Correspondencia: Lasty Balseiro Almario.

E-mail: lastybals@gmail.com 